\title{
ATTRIBUTE FUSION IN A LATENT PROCESS MODEL FOR TIME SERIES OF GRAPHS
}

\author{
Carey E. Priebe, Nam H. Lee, Youngser Park, Minh Tang \\ Johns Hopkins University \\ Department of Applied Mathematics and Statistics \\ Baltimore, Maryland 21218-2682 USA
}

\begin{abstract}
We consider anomaly/change point detection given a time series of graphs with categorical attributes on the edges. Various attributed graph invariants are considered, and their power for detection as a function of a linear fusion parameter is presented.
\end{abstract}

Index Terms - Anomaly Detection, Attributed Random Graphs, Fusion, Random Dot Product Graphs

\section{TIME SERIES OF ATTRIBUTED GRAPHS}

Given a time series of attributed graphs $G(t)=(V, \varphi(\cdot, t))$, $t=1,2, \cdots$, where the vertex set $V=[n]=\{1, \cdots, n\}$ is fixed throughout and edge attribution functions $\varphi(\cdot, t)$ : $\left(\begin{array}{l}V \\ 2\end{array}\right) \rightarrow\{0, \cdots, K\}$ are time-dependent, we wish to detect anomalies and/or change points. Let us consider vertices to represent "actors," and an edge between vertex $u$ and vertex $v$ at time $t(u v \in E(t)$, where the edge set $E(t)$ is given by $\left.E(t)=\left\{u v \in\left(\begin{array}{c}V \\ 2\end{array}\right): \varphi(u v, t)>0\right\}\right)$ represents the existence of a communications event between actors $u$ and $v$ during the time period $(t-1, t]$. Categorical edge attributes $\varphi(u v, t)$, when non-zero, represent some mode of the communication event between actors $u$ and $v$ during $(t-1, t]$; for instance, a topic label derived from the content of the communication. We will not consider directed edges or hypergraphs (hyper-edges consisting of more than two vertices) or multi-graphs (more than one edge between any two vertices at any time $t$ ) or self-loops (an edge from a vertex to itself) or weighted edges, although all of these generalizations of simple attributed graphs may be relevant for specific applications.

The specific anomaly we will consider is the "chatter" alternative - a small (unspecified) subset of vertices with altered communication behavior during some time period in an otherwise stationary setting, as depicted in Figure 1. This figure notionally depicts the entire vertex set $V$ behaving in some null state for $t=1, \cdots, t^{*}-1$; then, at time $t^{*}$, a collection of vertices $V_{A} \subset V\left(\left|V_{A}\right|=m, 2 \leq m \ll n\right)$ exhibits probabilistically altered behavior. (The remaining vertices remain

This work is supported in part by the Johns Hopkins University Human Language Technology Center of Excellence and a National Security Science and Engineering Faculty Fellowship (NSSEFF) award. in their null state throughout.) Our statistical inference task is to determine whether or not there has emerged a chatter group at some time $t=t^{*}$.

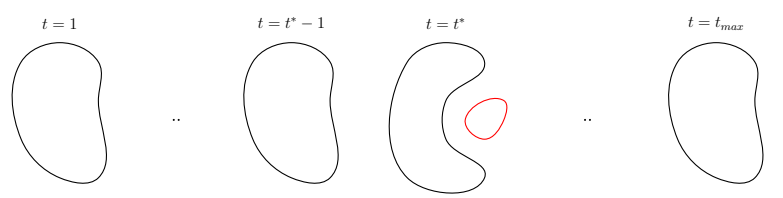

Fig. 1. Notional depiction of a time series of graphs in which the entire vertex set $V$ behaves in some null state for $t=$ $1, \cdots, t^{*}-1$ and then, at time $t^{*}$, a subset of vertices $V_{A}$ exhibits a change in behavior.

The latent process model for time series of attributed graphs presented in [1] provides for precisely this temporal structure. Each vertex is governed by a continuous time, finite state stochastic process $\left\{X_{v}(t)\right\}_{v \in V}$, with the state-space given by $\{0,1, \cdots, K\}$. The probability of edge $u v$ at time $t$ is determined by the inner product of the sub-probability vectors specified by $p_{w, k}(t)=$ $\int_{t-1}^{t} I\left\{X_{w}(\tau)=k\right\} d \tau, k=1, \cdots, K$, for $w=u, v$. The attribute $\varphi(u v, t)$ for edge $u v$ at time $t$, given that there is indeed an edge, is given by $P[\varphi(u v, t)=k \mid \varphi(u v, t)>$ $0]=p_{u, k}(t) p_{v, k}(t) / \sum_{\kappa=1}^{K} p_{u, \kappa}(t) p_{v, \kappa}(t)$. For the scenario depicted in Figure 1, the vertex processes $\left\{X_{v}(t)\right\}_{v \in V_{A}}$ are stationary until time $t^{*}-1$ and then undergo a change point, while the processes $\left\{X_{v}(t)\right\}_{v \in V \backslash V_{A}}$ remain stationary throughout all time.

Our latent process model produces a dependent time series of attributed graphs $G(t)$, each of which is itself a latent position model with conditionally independent edges given $\left\{X_{v}(\tau)\right\}_{v \in V, \tau \leq t}$. The model allows two simplifying approximations; a second-order (central limit theorem) approximation with temporally independent attributed random graphs each of which is itself a random dot product ([2],[3], and [4] Section 16.4) latent position model ([5], and [6] Section 3 ), and a first-order (law of large numbers) approximation with temporally independent attributed random graphs each of which is itself an independent edge random graph model [7]. 
The simplicity of the first-order approximation, depicted in Figure 2 for the special case of the kidney-egg stochastic blockmodel structure considered herein for the anomaly, provides a useful framework for description. If the vertex processes $\left\{X_{v}(t)\right\}_{v \in V}$ are independent and identical, with stationary probability vector $\pi_{0}=\left[\pi_{0,0}, \pi_{0,1}, \cdots, \pi_{0, K}\right]^{\prime}$, then the first-order approximation produces a temporally independent series of homogeneous independent edge attributed random graphs with $P[\varphi(u v, t)>0]=\left\langle\bar{\pi}_{0}, \bar{\pi}_{0}\right\rangle$ and $P[\varphi(u v, t)=k \mid \varphi(u v, t)>0]=\pi_{0, k}^{2} /\left\langle\bar{\pi}_{0}, \bar{\pi}_{0}\right\rangle$, where $\bar{\pi}_{0}=\left[\pi_{0,1}, \cdots, \pi_{0, K}\right]^{\prime}$. The vertex processes $\left\{X_{v}(t)\right\}_{v \in V_{A}}$ change at time $t^{*}-1$, taking on stationary probability vector $\pi_{A}$, so that $G\left(t^{*}\right)$ is a kidney-egg independent edge random graph with attribute probabilities defined using $\bar{\pi}_{0}$ and $\bar{\pi}_{0}$ for $u, v \in V \backslash V_{A}, \bar{\pi}_{0}$ and $\bar{\pi}_{A}$ for $u \in V \backslash V_{A}, v \in V_{A}$, and $\bar{\pi}_{A}$ and $\bar{\pi}_{A}$ for $u, v \in V_{A}$.

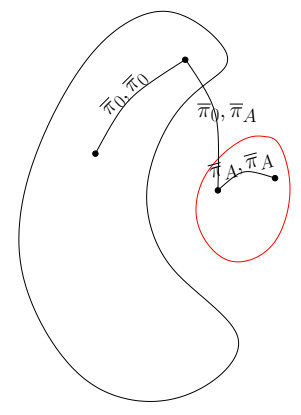

Fig. 2. An illustration of the kidney-egg stochastic blockmodel structure for $G\left(t^{*}\right)$. Edge probabilities (and their attributes) depend on the underlying vertex processes. For the first-order approximation, edges are governed by the stationary probability vectors.

One Monte Carlo replicate of this model yields, at time $t^{*}$, the latent position vectors presented in Figure 3 .

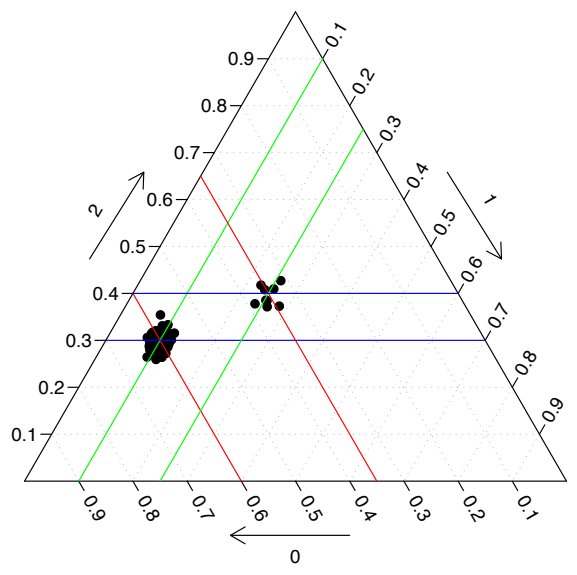

Fig. 3. Example of the vectors $\left\{X_{v}\right\}_{v \in V}$ in the simplex at time $t^{*}$ for our latent process model using parameters presented in Section 3 with $r=1024$.

\section{INVARIANTS}

In [8] the scan statistic graph invariants are introduced and applied to the problem of detecting chatter anomalies in time series of Enron graphs. In [9] various graph invariants (size, maximum degree, scan statistic, etc.) are considered for their power as test statistics and it is demonstrated via Monte Carlo that no single invariant is uniformly most powerful, while in [10] it is demonstrated that asymptotics can provide misleading comparative power analysis for size vs. maximum degree except for astronomically large graphs; see also [11] for a summary. In [12] fusion of various invariants is considered, and fusion of graph features and content is considered via theory and application in [13] and [14], respectively.

In this paper, we consider the problem of detecting chatter anomalies in time series of graphs using attributed invariants. Specifically, we consider linear attribute fusion with parameter $\lambda \in \mathbb{R}^{K}$ via

$$
\begin{gathered}
\operatorname{size}_{\lambda}(G(t))=\sum_{k=1}^{K} \lambda_{k} \sum_{u v} I\{\varphi(u v, t)=k\}, \\
\max _{\lambda}(G(t))=\max _{v} \sum_{k=1}^{K} \lambda_{k} \sum_{u \in N[v]} I\{\varphi(u v, t)=k\},
\end{gathered}
$$

and

$$
\operatorname{scan}_{\lambda}(G(t))=\max _{v} \sum_{k=1}^{K} \lambda_{k} \sum_{u, w \in N[v]} I\{\varphi(u w, t)=k\},
$$

where $N[v]=\{u: u v \in E(t)\} \cup\{v\}$ is the closed neighborhood of vertex $v$ in graph $G(t)$.

As described in [8] and [12], the invariants are standardized based on the recent past (see Figure 4).

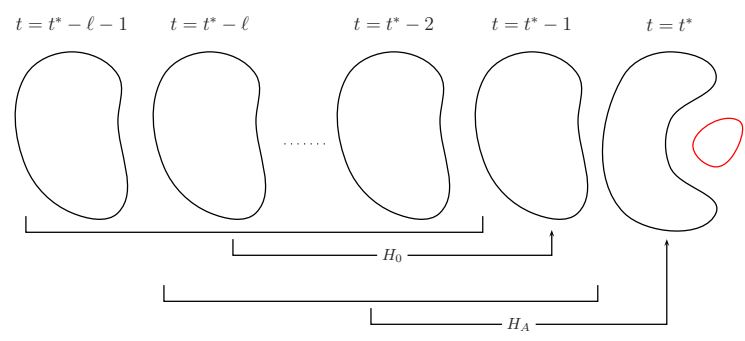

Fig. 4. Temporal standardization: when testing for change at time $t$, the recent past (graphs $G(t-\ell), \cdots, G(t-1)$ ) is used to standardize the invariants.

We present experimental results for anomaly detection on time series of simulated data from the model in [1], and demonstrate that optimal attribute fusion depends on invariant. 


\section{EXAMPLE}

We present here a simple yet illustrative Monte Carlo experiment using the following parameters:

$$
K=2, n=100, m=9, \ell=10,
$$

with stationary probability vectors

$$
\pi_{0}=[0.60,0.10,0.30]^{\prime}, \pi_{A}=[0.35,0.25,0.40]^{\prime},
$$

and transition matrices

$$
\begin{aligned}
Q_{0} / r & =\left[\begin{array}{ccc}
-2 / 3 & 1 / 6 & 1 / 2 \\
1 & -1 & 0 \\
1 & 0 & -1
\end{array}\right], \\
Q_{A} / r & =\left[\begin{array}{ccc}
-13 / 7 & 5 / 7 & 8 / 7 \\
1 & -1 & 0 \\
1 & 0 & -1
\end{array}\right] .
\end{aligned}
$$

(See [1] for a discussion of the vertex process rate parameter $r$. In summary, as $r \rightarrow \infty$ the second approximation (large $r$ ) and the first approximation (limiting $r$ ) obtain.)

Power estimates for our three attribute fusion statistics for this example are presented in Figures 5 and 6. We consider first approximation, second approximation, and exact model power estimates obtained via Monte Carlo simulation. Figure 5 shows power as a function of the vertex process rate parameter $r$. Figure 6 shows power as a function of angle $\theta$, where $\lambda=(\cos (\theta), \sin (\theta))$.

\section{CONCLUSIONS}

One notable implication of this work, inferred from Figure 5 , is that inferential performance in the mathematically tractable first- and second-order approximation models does indeed provide guidance for methodological choices applicable to the exact (realistic but intractable) model; furthermore, to the extent that the exact model is realistic, we may tentatively conclude that approximation model investigations have some bearing on real data applications.

Our main result regarding linear attribute fusion is that the optimal linear fusion parameter depends on the invariant considered. In particular, the results depicted in Figure 6 yield $\widehat{\theta}_{\text {size }}^{*}=0.22$ (compared to the theoretical $\theta_{\text {size }}^{*} \approx 0.24$ ), $\widehat{\theta}_{\text {maxd }}^{*}=0.31$, and $\widehat{\theta}_{\text {scan }}^{*}=0.15$. These optimal fusion parameter differences are statistically significant; combining this result with the "no uniformly most powerful invariant" result, we conclude that optimal linear attribute fusion theory requires significant additional development. Toward this end, the approximation models from [1] promise to be of assistance.

In addition to the social network analysis scenario considered herein for illustration (wherein vertices represent individual actors or organizations), hypothesis testing on time series of attributed graphs has application in areas as diverse as

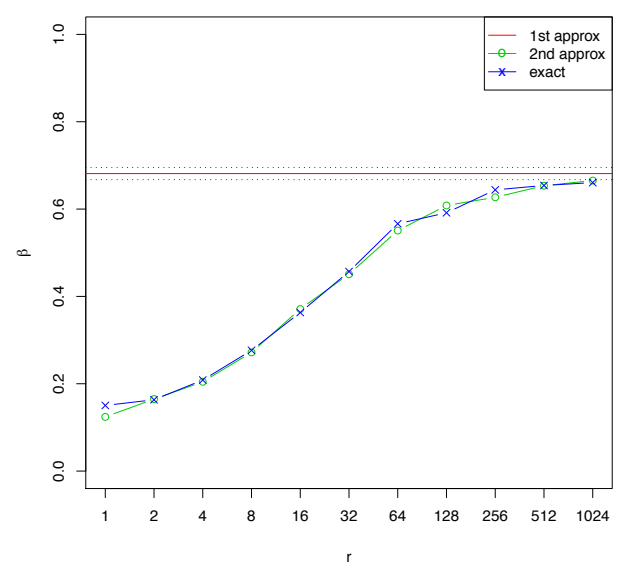

(a) $\operatorname{size}_{\lambda}$

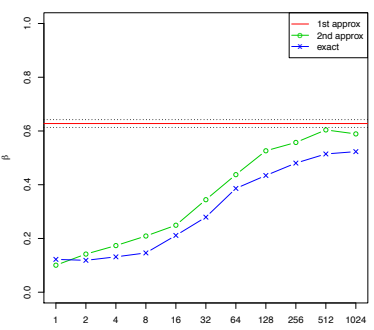

(b) $\max d_{\lambda}$

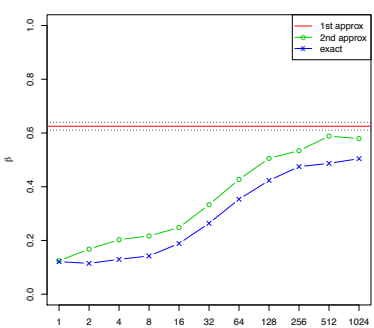

(c) $\operatorname{scan}_{\lambda}$
Fig. 5. Power $\beta\left(\theta_{r}^{*}, r\right)$ at $\theta_{r}^{*}=\arg \max _{\theta} \beta(\theta, r)$ as a function of $r$ for the three invariants at test size $\alpha=0.05$. (10000 Monte Carlo replicates yields $\sigma_{\widehat{\beta}} \leq 0.005$.) The horizontal line represents first-order approximation \pm three standard deviations, and the two curves represent the second approximation and exact model. The second approximation results match well with the exact model results, and both match well for large $r$ with the first-order approximation results.

connectome inference (wherein vertices are neurons or brain regions) and text processing (wherein vertices represent authors or documents). These applications, and others, may benefit from generalization to directed, hyper, multi, loopy, weighted graphs, as well as the consideration of inference with errorful attributes through an attribute confusion matrix.

\section{REFERENCES}

[1] N. H. Lee and C. E. Priebe, "A Latent Process Model for Time Series of Attributed Random Graphs," Statistical Inference for Stochastic Processes, forthcoming.

[2] E. R. Scheinerman and K. Tucker, "Modeling Graphs 
Using Dot Product Representations," Computational Statistics, vol. 25, pp. 1-16, January 2010.

[3] S. J. Young and E. R. Scheinerman, "Random Dot Product Graph Models for Social Networks," Proc. 5th ICAM, pp. 138-149, 2007.

[4] B. Bollobás, S. Janson, and O. Riordan, "The Phase Transition in Inhomogeneous Random Graphs," Random Structures and Algorithm, vol. 31, pp. 3-122, 2007.

[5] P. Hoff, A. E. Raftery, and M. S. Handcock, "Latent Space Approaches to Social Network Analysis," Journal of the American Statistical Association, vol. 97, pp. 1090-1098, 2002.

[6] A. Goldenberg, A. X. Zheng, S. E. Fienberg, and E. M. Airoldi, "A survey of statistical network models," Foundations and Trends in Machine Learning, vol. 2, no. 2, pp. 129-233, 2009.

[7] B. Bollobás, Random Graphs, Cambridge University Press, 2nd edition, 2001.

[8] C. E. Priebe, J. M. Conroy, D. J. Marchette, and Y. Park, "Scan Statistics on Enron Graphs," Computational and Mathematical Organization Theory, vol. 11, pp. 229247, October 2005.

[9] H. Pao, G. A. Coppersmith, and C. E. Priebe, "Statistical Inference on Random Graphs: Comparative Power Analyses via Monte Carlo," Journal of Computational and Graphical Statistics, forthcoming.

[10] A. Rukhin and C. E. Priebe, "A comparative power analysis of the maximum degree and size invariants for random graph inference," Journal of Statistical Planning and Inference, forthcoming.

[11] C. E. Priebe, G. A. Coppersmith, and A. Rukhin, "You Say Graph Invariant, I Say Test Statistic," ASA Sections on Statistical Computing Statistical Graphics SCGN Newsletter, vol. 21, no. 2, December 2010.

[12] Y. Park, C.E. Priebe, and A. Youssef, "Anomaly detection in time series of graphs using fusion of invariants," submitted for publication.

[13] J. Grothendieck, C. E. Priebe, and A. L. Gorin, "Statistical Inference on Attributed Random Graphs: Fusion of Graph Features and Content," Computational Statistics and Data Analysis, vol. 54, pp. 1777-1790, 2010.

[14] C. E. Priebe, Y. Park, D. J. Marchette, J. M. Conroy, J. Grothendieck, and A.L. Gorin, "Statistical Inference on Attributed Random Graphs: Fusion of Graph Features and Content: An Experiment on Time Series of Enron Graphs," Computational Statistics and Data Analysis, vol. 54, pp. 1766-1776, 2010.

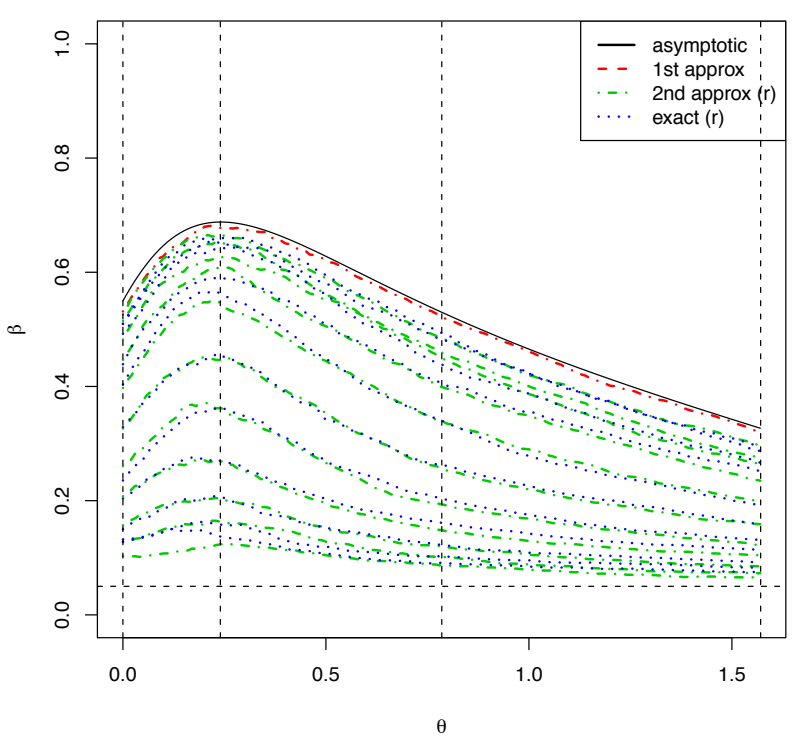

(a) $\operatorname{size}_{\lambda}$

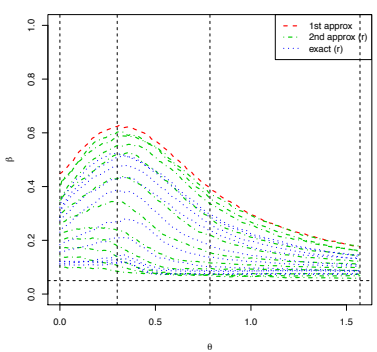

(b) $\max d_{\lambda}$

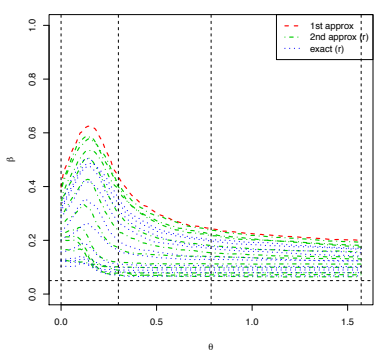

(c) $\operatorname{scan}_{\lambda}$
Fig. 6. Power $\beta$ as a function of angle $\theta$ for $\lambda=$ $(\cos (\theta), \sin (\theta))$. The plot shows analytic asymptotic result (black, available for size only), and first approximation (red), second approximation (green), and exact model (blue) estimates for the various invariants via Monte Carlo. (10000 Monte Carlo replicates yields $\sigma_{\widehat{\beta}} \leq 0.005$.) (Horizontal line is $\alpha=0.05$.) The four vertical lines correspond to $\theta \in\left\{0, \pi / 2, \pi / 4, \theta_{\text {size }}^{*} \approx 0.24\right\}$, which correspond to attribute weighting schemes of: consider only attribute 1 , consider only attribute 2 , consider both attributes equally, and the attribute weight ratio of approximately 3.7:1. (The asymptotic first-order approximation power analysis for size $_{\lambda}$ in [1] yields the analytic maximizer $\theta_{\text {size }}^{*} \approx 0.24$; this corresponds to an attribute weight ratio for optimal power of approximately 3.7:1.) The second approximation results match well with the exact model results, and both match well for large $r$ with the first-order approximation results. The opitmal $\theta$ is apparently different for the three different invariants. (Because we are considering a one-sided test, rejecting for large values of our statistics, and because $\pi_{A, k}>\pi_{0, k}$ for both $k=1,2$, the power is maximized in the first quadrant.) 